\title{
Tracking network state from combined SCADA and synchronized phasor measurements
}

\author{
Mevludin Glavic Thierry Van Cutsem* \\ Dept. of Electrical Engineering and Computer Science (Montefiore Institute), \\ University of Liège, Sart Tilman B37, B-4000 Liège, Belgium. \\ * Fund for Scientific Research - FNRS.
}

\begin{abstract}
Initiated by the authors' previous work on state reconstruction from a limited number of synchrophasors [1], this paper goes one step further by exploring the possibility to track the network state using both SCADA and synchronized phasor measurements. When a SCADA measurement is received, it is used in the next state reconstruction; otherwise, it is replaced by a pseudo-measurement stemming from the previous state reconstruction. The approach resorts to a standard weighted least squares formulation and Hachtel's augmented matrix method. State reconstruction is intended to be used at a much higher rate than classical state estimation, for instance every second. It has been validated using simulated measurements obtained from detailed time simulation.
\end{abstract}

\section{Introduction}

The emergence of the time synchronized phasor measurement technology opens new perspectives to design advanced power system monitoring schemes [2]. Enhancement of state estimation is a natural application of this technology [2], [3] and previous publications dealt with the improvement of various aspects of state estimation such as accuracy, identification of topological and parameter errors, bad data processing, etc. [3]. For practical reasons, it may be advantageous to leave existing Weighted Least Squares (WLS) state estimator software unchanged and exploit synchronized phasor measurements in a pre- or post-processing step [4], [5].

If synchrophasors are available in a sufficient number to ensure full system observability, then a linear problem can be formulated in static state estimation [2] while Kalman filter can be used to track the network state [6] or system dynamics [7]. However, the wider deployment of Phasor Measurement Units (PMUs) must be supported by adequate upgrades of communication infrastructures and is considerably hampered by the lack of this support. Consequently, existing PMU configurations are far from ensuring full system observability. Taking advantage of scarce synchrophasor measurements will thus remain a challenge in effective exploitation of existing PMU configurations.

Motivated by this challenge, the authors proposed recently a method to reconstruct and track the network state from a limited number of PMU data. The method is aimed at being used in between standard state estimation runs. Tracking the system evolution at higher rate could bring useful information, increase system situational awareness [8], [9] and contribute to decreasing the probability of blackout by lack of real-time information [10].

The idea of computing updated states between two accurate solutions of state estimator can be traced back to [11], in which a two-level approach was proposed in the context of least absolute value state estimation. Accurate solutions were computed at the rate of classical state estimation while approximate solutions were obtained by solving generalized power flows at SCADA rate. Based on classical state estimation residual analysis, a subset of $n$ measurements was identified in order to determine the $n$ unknowns, repetitively between two accurate state estimations.

The concept proposed by the authors in [1], [12] is close to that of a tracking state estimator [13], [14], as originally formulated in (the discussion accompanying) [14], since it does not rely on a dynamic system model or approximate dynamics of system state. The method relies on synchronized phasor measurements together with power and voltage pseudomeasurements obtained from the previous state reconstruction, in a recursive manner.

This paper extends the work in [1], [12] with the aim to reconstruct coherent network states from multi-rated measurements, namely a limited number of PMU data together with traditional SCADA measurements, at a rate close to that of the SCADA system, i.e. much higher than classical state estimation. To this purpose, the state reconstruction problem is reformulated in order to process SCADA measurements, immediately after they have been received by the real-time system. In the state reconstruction runs where a new value has not been received for a given measurement, a pseudomeasurement is used instead, which is obtained from the previous state reconstruction.

In other words, what is proposed in this paper is an integrated technique to take the largest possible benefit from all SCADA and PMU data, with the aim of tracking system evolution at a much higher rate than present-day control center state estimators. The approach is scalable: it accommodates scarce PMU configuration while higher accuracy will be obtained from the future, richer PMU configurations. 


\section{Time delays affecting SCADA measurements}

As is well known, SCADA measurements are not synchronized. Figure 1 outlines the typical process of gathering measurements through Remote Terminal Units (RTUs), and the associated time delays [15].

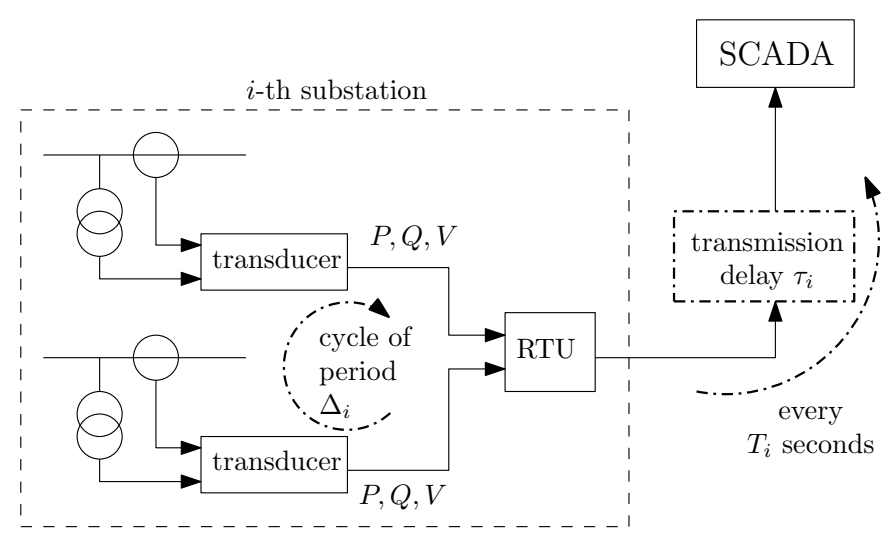

Fig. 1. SCADA measurement gathering process

In a given substation, voltage magnitude, active and reactive power flow (and occasionally, current) measurements are collected sequentially. More precisely, the various measurement devices (transducers) are "visited" cyclically and the measured values are stored sequentially in the memory of the RTU. For the $i$-th substation, the period of this cyclic measurement gathering is denoted by $\Delta_{i}$. Its order of magnitude is one second.

At a given time $t^{\star}$, the RTU of the $i$-th substation sends the contents of its memory to the SCADA system of the control center. When this takes place, the $j$-th measured value present in the memory refers to a previous time $t^{\star}-\delta_{j i}$ where $\delta_{j i}$ is a value in the interval $\left[0, \Delta_{i}\right]$.

It takes some time $\tau_{i}$ for the set of data to be received by the control center. This transmission delay is typically below half a second. In the sequel, it is assumed for simplicity that the measurements of a given substation are received all together.

While measurements are gathered continuously within a substation, the RTU communicates with the SCADA system of the control center at regular time intervals only. The time between two successive communications is denoted by $T_{i}$ for the $i$-th substation. $T_{i}$ may lie in between two and five seconds, depending on the technology, the age of the equipment, etc.

The various delays are further illustrated in Fig. 2, for a hypothetical case of two substations each provided with two measurements. In that figure, a cross indicates the time at which a measurement is taken, and the following circle the time at which it is received by the SCADA system.

The figure also sketches how measurements are processed by a (static) State Estimator (SE). The SE uses the last available SCADA values of the various measurements, and treats them

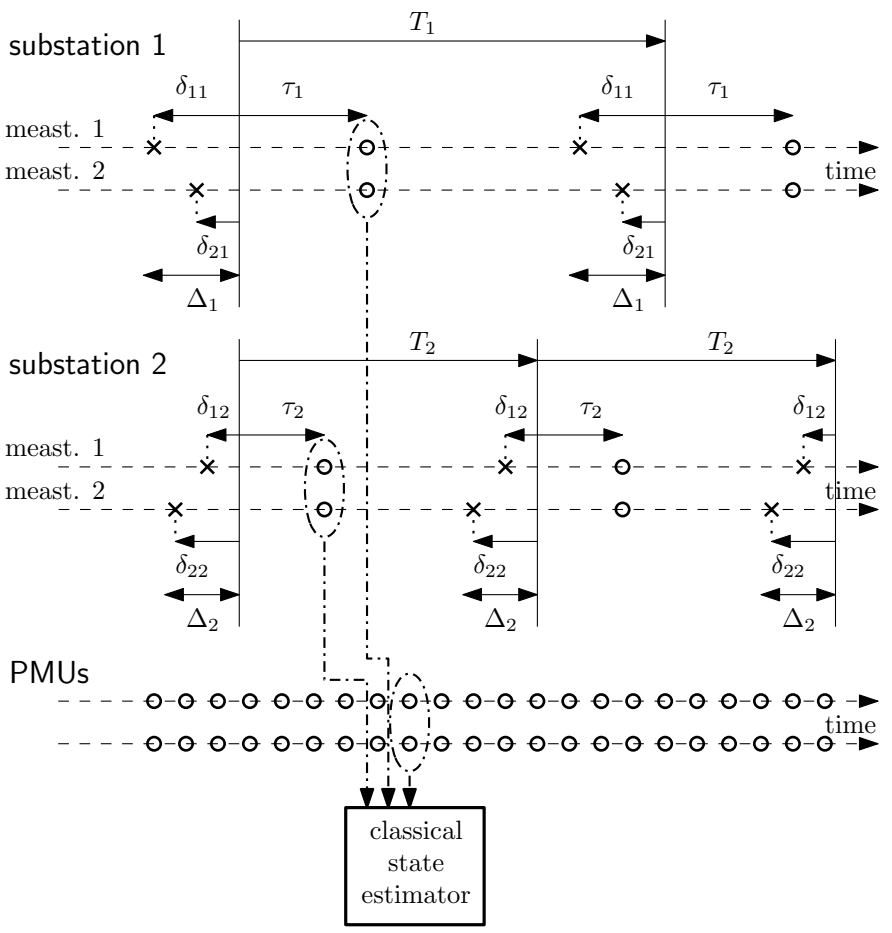

Fig. 2. Delays associated with data gathering and processing by classical state estimator. A $\mathrm{x}$ indicates the time at which a measurement is taken; a 0 the time at which it is received by the SCADA system

as if they had been taken at the same time, while they are affected by time skew. This makes the estimates unreliable in case of significant transients. If PMU data are available in addition to SCADA, they potentially offer the possibility to handle such transients. However, in the worst case, their treatment together with the SCADA measurements may cause them to be flagged as bad data by the SE.

While PMU data are also affected by transmission delays of the type $\tau_{i}$ (introduced by phasor data concentrators and communication network [2]), they are synchronized and timetagged. Hence it is always possible, in principle, to process synchronized phasor measurements all relative to the same time in the recent past, and relate the reconstructed state to that time. Hence, for simplicity, no delay will be considered for synchrophasors in the remaining of this paper.

\section{Objective and principle of the proposed method}

\section{Overall objective}

The overall objective of the proposed method is to track the changing network state by reconstructing the vector of complex bus voltages every $T_{r}$ seconds. The period $T_{r}$ is intended to be much smaller than the time span between two successive classical SE runs, which is in the order of one to several minutes. Even more, the period $T_{r}$ is intended to be smaller than the refreshing rate of SCADA measurements, i.e. $T_{r}<T_{i}, \forall i$. 
To this purpose, synchronized phasor measurements are used. The latter are available at a rate of 10 to 120 samples per second, typically [2]. At the current stage of development of the method, it is not intended to perform state reconstruction at such a high rate. Hence, only a subset of the successive synchronized phasor measurement snapshots is going to be used.

In the sequel, $T_{r}$ has been taken equal to one second. Shorter values, for instance $T_{r}=0.1 \mathrm{~s}$, could be considered, as in [1].

\section{Principle}

The following are the ideas underlying the proposed method:

1) at time $t$, the network state is reconstructed using the corresponding synchronized (bus voltage and branch current) phasor measurements as well as the new (voltage magnitude, active or reactive power) SCADA measurements that have been received since the last state reconstruction, i.e. in the time interval $\left[t-T_{r}, t[\right.$. SCADA measurements received before time $t-T_{r}$ are no longer used to minimize the already mentioned time skew;

2) the limited number of those synchrophasor and SCADA measurements make the system unobservable. Pseudomeasurements must be added to restore observability;

3) a pseudo-measurement is used in replacement for each SCADA measurement not involved in the current state reconstruction;

4) the value used at time $t$ for that pseudo-measurement is the corresponding value calculated from the last reconstructed state vector, i.e. at time $t-T_{r}$;

The procedure is shown graphically in Fig. 3, matching the example of Fig. 2. Circles have the same meaning as in Fig. 2, while black squares denote pseudo-measurements obtained from previous runs of the state reconstruction algorithm. Each successive state reconstruction uses a measurement vector of constant dimension, including synchronized phasor, SCADA and pseudo-measurements. When a SCADA measurement is received, it is used in the next state reconstruction; otherwise, it is replaced by the value stemming from the previous state reconstruction. This recursive processing is inspired of [1].

The SCADA measurements used at time $t$ come with delays $\delta_{j i}+\tau_{i}+\epsilon_{i}$ where $\delta_{j i}$ and $\tau_{i}$ have been already defined, and $\epsilon_{i}$ is the dead time between the arrival of measurements from substation $i$ and their processing, as shown in Fig. 3. Hence, even if they resort to the same sensors as PMUs, these SCADA measurements are intrinsically less accurate, and must be assigned a lower weight in state reconstruction. Pseudomeasurements must also be assigned a lower weight compared to synchronized phasor measurements.

\section{Solution algorithm}

The synchronized phasor, SCADA and pseudo-measurements are processed by a standard WLS estimation under constraints.

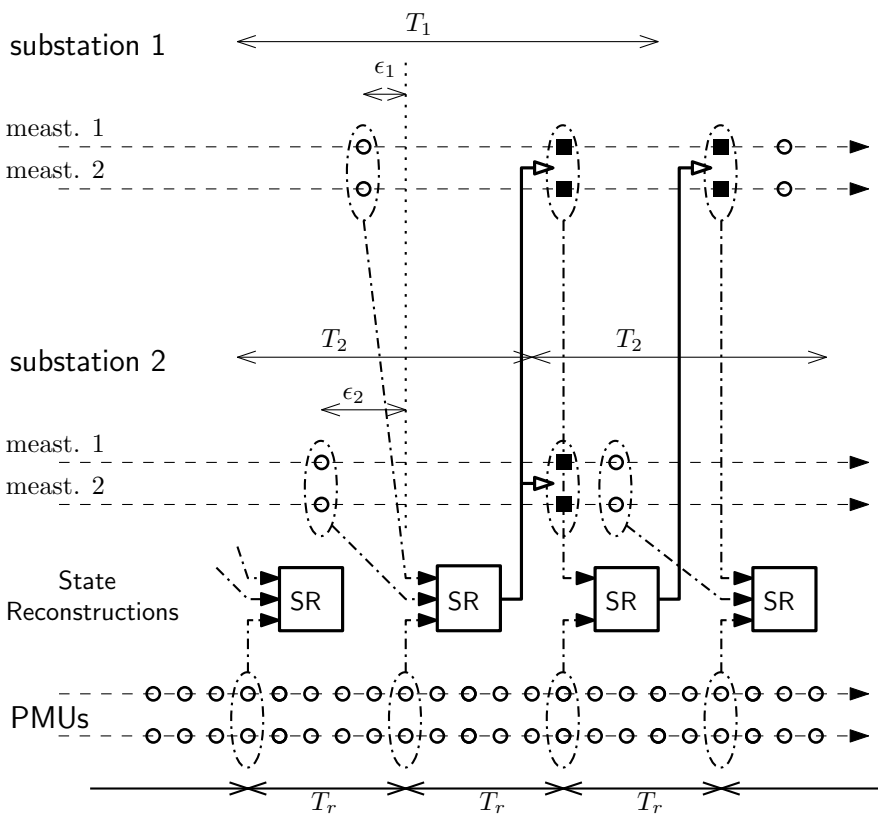

Fig. 3. Using synchronized phasor, SCADA and pseudo-measurements for state reconstruction. A $\bigcirc$ indicates the time at which a measurement is received by the SCADA system; a denotes a pseudo-measurement

Thus, a variety of methods can be used such as normal equations, normal equations with equality constraints, or Hachtel's augmented matrix [16], [17], [18]. The latter method has shown very good performances in the application of concern here. Its formulation is briefly recalled hereafter, and its results will be presented in the sequel.

A Cartesian component formulation is used. The state vector $\boldsymbol{x}$ is thus composed of the real and imaginary parts of the complex bus voltages. This leads to a linear model $\boldsymbol{h}(\boldsymbol{x})$ for the synchrophasor part of the measurement vector $z$.

The objective is to minimize the sum of squared residuals $r$ weighted by the matrix $W$ :

$$
\min _{\boldsymbol{x}, \boldsymbol{r}} \frac{1}{2} \boldsymbol{r}^{T} \mathbf{W} \boldsymbol{r}
$$

subject to equality constraints corresponding to the zero injections at transit buses:

$$
f(x)=0
$$

as well as the measurement residual definition:

$$
\boldsymbol{r}-\boldsymbol{z}+\boldsymbol{h}(x)=\mathbf{0}
$$

Forming the Lagrangian $\mathcal{L}$ and expressing the first-order optimality conditions yields:

$$
\begin{array}{lrl}
\frac{\partial \mathcal{L}}{\partial \boldsymbol{r}}=\mathbf{0} \Leftrightarrow & \boldsymbol{W r}-\boldsymbol{\mu}=\mathbf{0} \\
\frac{\partial \mathcal{L}}{\partial \boldsymbol{\mu}}=\mathbf{0} \Leftrightarrow & \boldsymbol{r}-\boldsymbol{z}+\boldsymbol{h}(\boldsymbol{x})=\mathbf{0} \\
\frac{\partial \mathcal{L}}{\partial \boldsymbol{x}}=\mathbf{0} \Leftrightarrow & -\boldsymbol{H}^{T}(\boldsymbol{x}) \boldsymbol{\mu}-\boldsymbol{F}^{T}(\boldsymbol{x}) \boldsymbol{\lambda}=\mathbf{0} \\
\frac{\partial \mathcal{L}}{\partial \boldsymbol{\lambda}}=\mathbf{0} \Leftrightarrow & \boldsymbol{f}(\boldsymbol{x})=\mathbf{0}
\end{array}
$$


where $\boldsymbol{\lambda}$ (resp. $\boldsymbol{\mu}$ ) is the vector of Lagrange multipliers relative to (2) (resp. (3)), and $\boldsymbol{H}$ (resp. $\boldsymbol{F}$ ) is the Jacobian matrix of $\boldsymbol{h}$ (resp. $\boldsymbol{f}$ ) with respect to $\boldsymbol{x}$. Assuming a nonsingular $\mathrm{W}$ matrix, $\boldsymbol{r}$ can be eliminated, to obtain the reduced set of equations:

$$
\begin{aligned}
\mathbf{W}^{-1} \boldsymbol{\mu}-\boldsymbol{z}+\boldsymbol{h}(x) & =\mathbf{0} \\
\boldsymbol{H}^{T}(\boldsymbol{x}) \boldsymbol{\mu}+\boldsymbol{F}^{T}(\boldsymbol{x}) \boldsymbol{\lambda} & =\mathbf{0} \\
\boldsymbol{f}(\boldsymbol{x}) & =\mathbf{0}
\end{aligned}
$$

Hachtel's method consists of considering $\boldsymbol{F}$ and $\boldsymbol{H}$ as constant matrices in (5b) and using Newton's method to deal with the nonlinear equations (5a) and (5c). This leads to solving, at the $k$-th iteration $(k=1,2, \ldots)$, the sparse, symmetric linear system :

$$
\left[\begin{array}{ccc}
\boldsymbol{W}^{-1} & \boldsymbol{H} & \mathbf{0} \\
\boldsymbol{H}^{T} & \mathbf{0} & \boldsymbol{F}^{T} \\
\mathbf{0} & \boldsymbol{F} & \mathbf{0}
\end{array}\right]\left[\begin{array}{c}
\boldsymbol{\mu}^{k} \\
\Delta \boldsymbol{x} \\
\boldsymbol{\lambda}^{k}
\end{array}\right]=\left[\begin{array}{c}
\boldsymbol{z}-\boldsymbol{h}\left(\boldsymbol{x}^{k-1}\right) \\
\mathbf{0} \\
-\boldsymbol{f}\left(\boldsymbol{x}^{k-1}\right)
\end{array}\right]
$$

and incrementing the state vector $\boldsymbol{x}$ according to:

$$
\boldsymbol{x}^{k}=\boldsymbol{x}^{k-1}+\Delta \boldsymbol{x}
$$

Note that the structure of the coefficient matrix in (6) does not change from one reconstruction to the next, unless there is a change in network topology. When a SCADA measurement is replaced by a pseudo-measurement in $\boldsymbol{z}$, the corresponding weight is adjusted in $\boldsymbol{W}$, if it is different, of course.

\section{Results: test system, events and measurements}

\section{Test system and events}

For easy comparison, simulation results are provided for the Nordic32 test system documented in [19]. The one-line diagram of this 74-bus, 102-branch, 20-machine system is shown in Fig. 4.

A detailed time simulation under phasor approximation has been performed to obtain the "exact" system evolution after a severe disturbance. The simulated model involves:

- detailed representation of the 20 synchronous generators;

- generic models for AVRs, excitation systems, prime movers and speed governors;

- Load Tap Changers (LTCs) controlling with various delays the voltages at 22 distribution buses (shown without number in Fig. 4) where loads are connected;

- exponential model for load power variation with voltage;

- OverExcitation Limiters (OELs) with either fixed- or inverse-time response.

In order to test the method in stringent conditions, the following sequence of events has been considered:

- three-phase fault applied on line 4032-4044 at $t=1 \mathrm{~s}$, and cleared by opening that line;

- progressive degradation of operating conditions due to longterm voltage instability caused by LTCs and OELs;

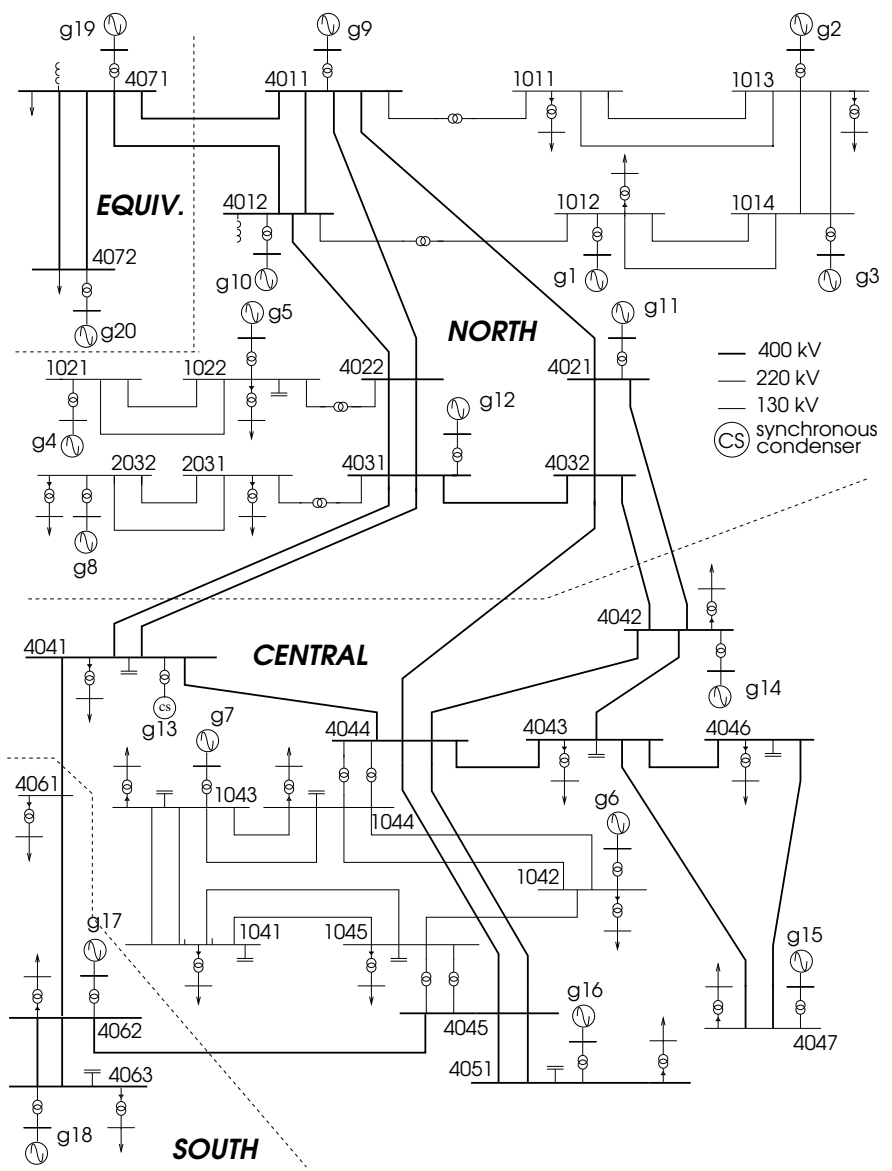

Fig. 4. Nordic-32 test system

- emergency system stabilization by under-voltage load shedding. Six blocks of load are shed, three at the MV bus connected to bus 1041 (at $t=116,135$ and $222 \mathrm{~s}$ ) and three at the MV bus connected to bus 1044 (at $t=135,171$ and $191 \mathrm{~s}$ ), for a total of $300 \mathrm{MW}$.

The model processed in state reconstruction involves 52 (transmission or generator) buses and 80 (line or transformer) branches. Distribution buses and transformers are not considered. The loads are thus the powers entering the distribution transformers. The tap changes in these transformers and the load curtailments are neither known nor modeled in state reconstruction. On the other hand, the transmission line tripping due to fault clearing is assumed to be known.

Ten buses have zero injections (namely buses 4011, 4012, 1014, 1021, 4022, 4021, 4031, 4032, 4044 and 4045).

\section{Simulation of synchrophasor measurements}

Three PMU configurations have been considered:

- Configuration 1: six PMUs located at buses g6, g7, g11, $\mathrm{g} 14, \mathrm{~g} 15$, and $\mathrm{g} 16$, providing twelve synchrophasors (six voltages and six currents). This configuration was identified in [1] as a good compromise between low number of PMUs 
and accuracy of state reconstruction in the Central region (see Fig. 4) affected by voltage instability. PMUs are located at generator buses and, hence, have two channels only, to avoid too rich a coverage of this small system;

- Configuration 2: includes two PMUs, as shown in Fig.5:

- one at bus 1043, providing one bus voltage and four branch current synchrophasors, and

- one at bus 4011, providing one voltage and six current synchrophasors.

This configuration was selected to have a better coverage by PMUs of buses 1041 and 1044, where load curtailment takes place;

- Configuration 3: includes two other PMUs:

- one at bus 4044, providing one voltage and six current synchrophasors, and

- one at bus 4011, as in Configuration 2.

This PMU configuration does not offer a good coverage of buses where load shedding takes place.
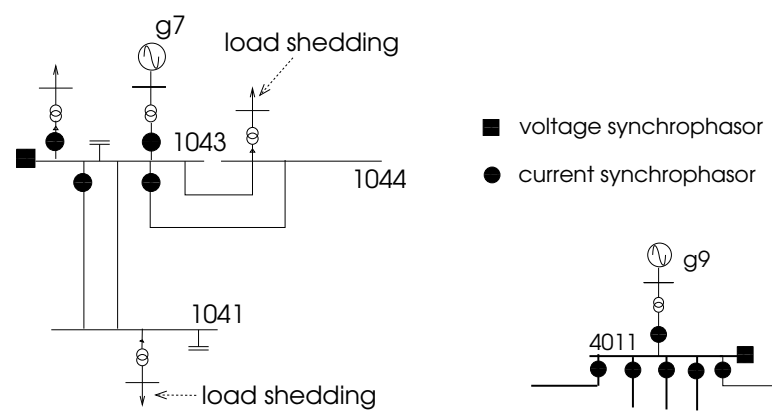

Fig. 5. PMU configuration No.2 at buses 1043 and 4011

PMU data have been obtained by sampling at regular time interval the rectangular components of voltages and currents given by time simulation, and adding to each component a Gaussian noise $N(0, \sigma)$ with $\sigma=0.002 \mathrm{pu}$. Hence, the simulated synchrophasor measurements are affected by sensor noise as well as by transients.

\section{Simulation of SCADA measurements}

The following 154 SCADA measurements have been considered: voltage magnitudes at the 20 generator buses, active and reactive power flows at one end of 67 branches. It has been assumed that the voltage and power measurements taken at a bus are transmitted all together to the SCADA system, every $T_{i}$ seconds, and with a transmission delay $\tau_{i} . T_{i}$ and $\tau_{i}$ vary from one bus to another, with $2 \leq T_{i} \leq 5 \mathrm{~s}$ and $0.1 \leq \tau_{i} \leq 0.5 \mathrm{~s}$. At a given bus, the measurements are collected at different instants, leading to a delay $\delta_{j i}$ which varies from one measurement to another, with values in the range $[0.1,0.9] \mathrm{s}$.

Figure 6 illustrates how SCADA measurements were generated and used with delays in state reconstruction.

The solid line shows (a portion of) the "exact" evolution of the active power flow in the 130-kV line 1043-1041. The transition at $t=116 \mathrm{~s}$ corresponds to load curtailment at bus 1041 .

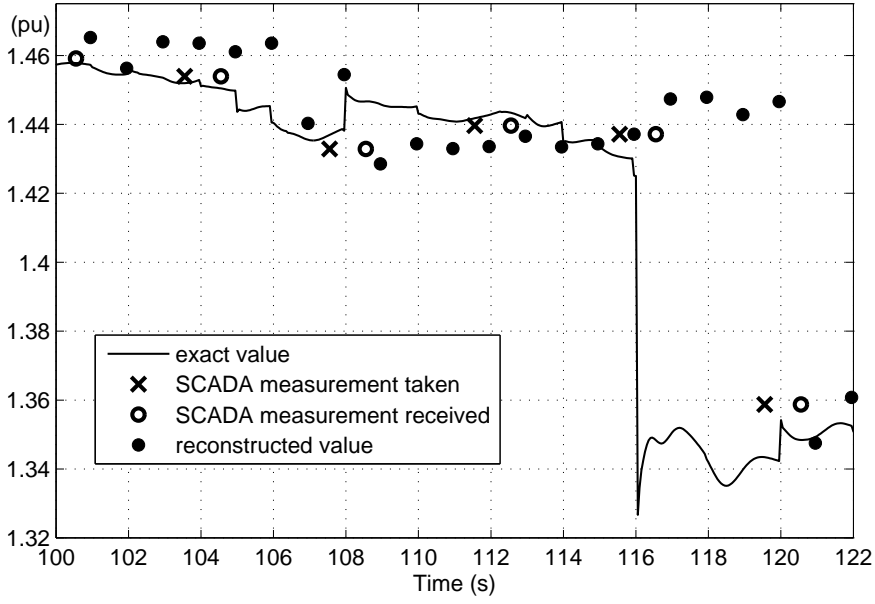

Fig. 6. Example of SCADA measurement generation and delayed exploitation

The crosses show the times and values of the successive measurements of that power flow. They depart from the solid line under the effect of measurement noise, simulated as Gaussian random variable $N(0, \sigma)$ with $\sigma=0.01 \mathrm{pu}=1$ MW/Mvar. Voltage measurements were simulated similarly, with $\sigma=0.002 \mathrm{pu}$. The circles show when the corresponding measurements were received by the SCADA system of the control center. In the case of Fig. 6, the delay $\delta_{j i}+\tau_{i}$ is a little larger than one second, while a measurement is sent every $T_{i}=4$ seconds.

The black disks show the corresponding reconstructed values. The period of state reconstruction is $T_{r}=1 \mathrm{~s}$. For instance, the SCADA measurement received at $t=108.5 \mathrm{~s}$ was used to reconstruct the state at $t=109 \mathrm{~s}$. The reconstructed power flow value at $t=109 \mathrm{~s}$ was used as pseudo-measurement in the reconstruction performed at $t=110 \mathrm{~s}$, and so on until $t=113 \mathrm{~s}$, at which time a new SCADA measurement is available.

Note that the measurement received at $t=116.5 \mathrm{~s}$ was taken before the load curtailment transition. This "outdated" value impacts the next four state reconstructions, until the SCADA measurement at $t=120.5 \mathrm{~s}$ brings information and allows state reconstruction to reset close to the exact evolution. This reset capability was not present in the method of Ref. [1], as illustrated later on.

\section{Measurement weights}

A diagonal weighting matrix $\mathbf{W}$ has been considered. The diagonal entries are of the form $1 / \sigma^{2}$, where $\sigma$ is the standard deviation of the corresponding measurement error [18]. The values of $\sigma$ are given in Table I. The standard deviation assigned to a SCADA measurement is larger than that of the corresponding measurement noise, to somewhat account for the delay $\delta_{j i}+\tau_{i}$. The standard deviations assumed for synchrophasors match those of the corresponding measurement noises. Pseudo-measurements are assigned the same weights as SCADA measurements, for simplicity. 
TABLE I

STANDARD DEVIATIONS ASSIGNED TO VARIOUS DATA

\begin{tabular}{|c|c|}
\hline measurement type & $\sigma(\mathrm{pu})$ \\
\hline \hline SCADA power flow & 0.100 \\
SCADA voltage & 0.006 \\
synchrophasor & 0.002 \\
pseudo-measurement & same as SCADA measurements \\
\hline
\end{tabular}

Further investigations would be needed to identify "optimal" weighting factors, or possibly correlations between measurements. Nevertheless, the results were found rather insensitive to these parameters; furthermore, the accuracy is satisfactory even with the above heuristic settings.

\section{Results: accuracy of state reconstruction}

All voltages (resp. powers) are in per unit on the nominal voltage (resp. 100-MVA) base.

\section{PMU configuration No. 1}

Figures 7 and 8 show the exact and reconstructed evolutions of respectively the voltage magnitude and the active power of the load at bus 1041. None of the two quantities is measured. Figure 9 shows the exact and reconstructed evolutions of the active power flow in line 1043-1041, which is provided with a SCADA measurement. As can be seen, the overall accuracy of state reconstruction is quite satisfactory, even after the transients due to load curtailment.

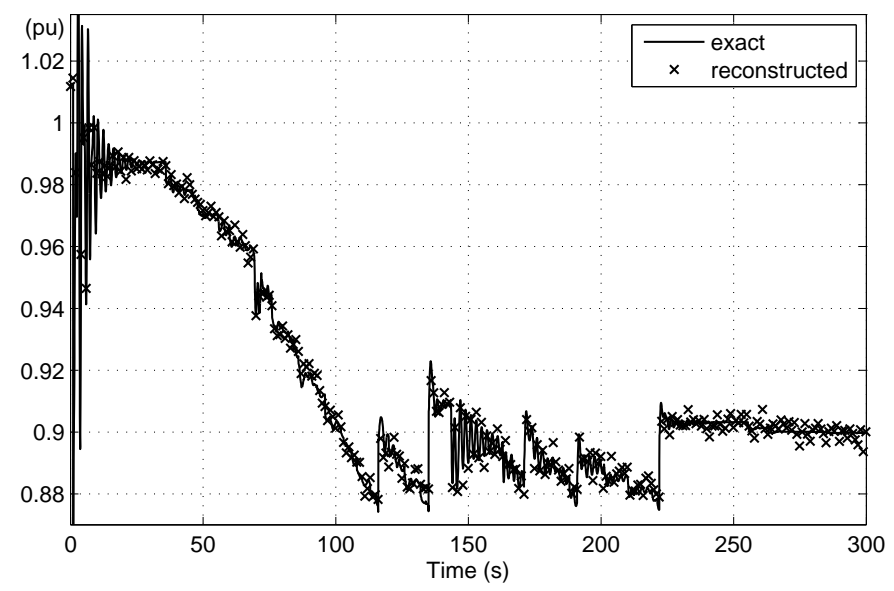

Fig. 7. Exact and reconstructed voltage magnitude at bus 1041 (PMU configuration No. 1)

Figure 10 shows the reconstructed load active power at the same bus 1041, obtained by the method of Ref. [1]. In the latter, SCADA measurements are not exploited, while the pseudo-measurements used to complement synchrophasors are the active and reactive powers at load buses and the active powers and voltages at generator buses. This choice of pseudomeasurements was based on the assumption that the corresponding quantities do not vary too much over time. Clearly, this assumption is invalidated in the scenario considered here, where load is curtailed. Synchrophasor measurements alone

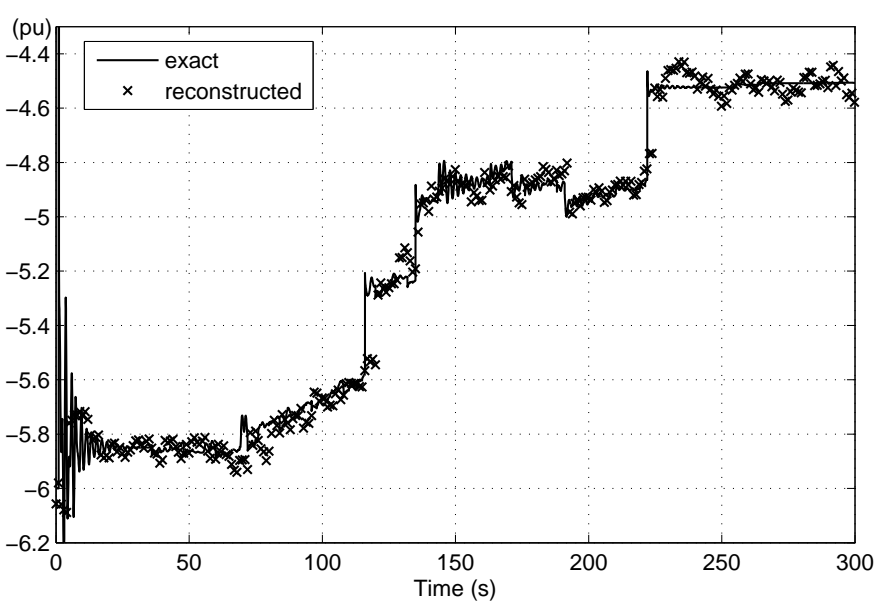

Fig. 8. Exact and reconstructed active power at bus 1041 (PMU configuration No. 1)

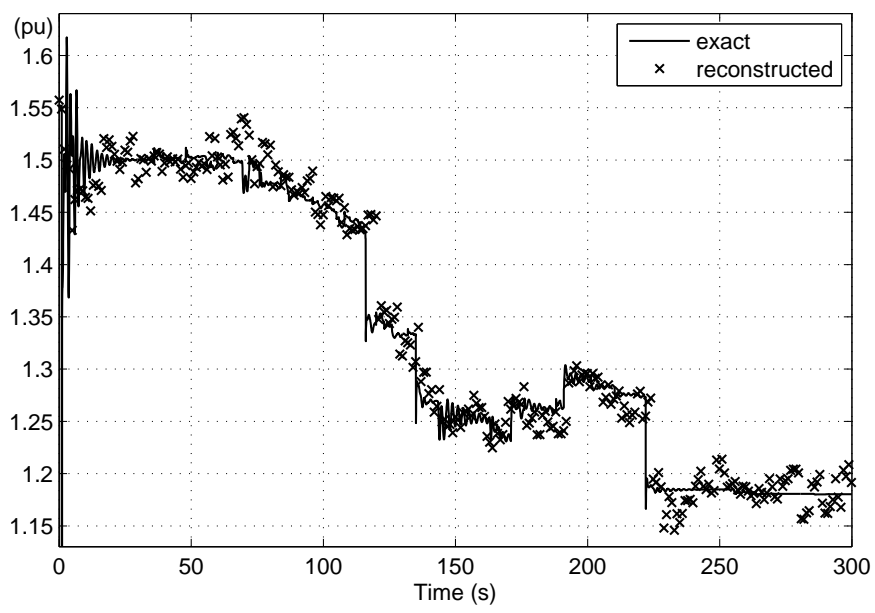

Fig. 9. Active power flow in line 1043-1041 (PMU configuration No. 1)

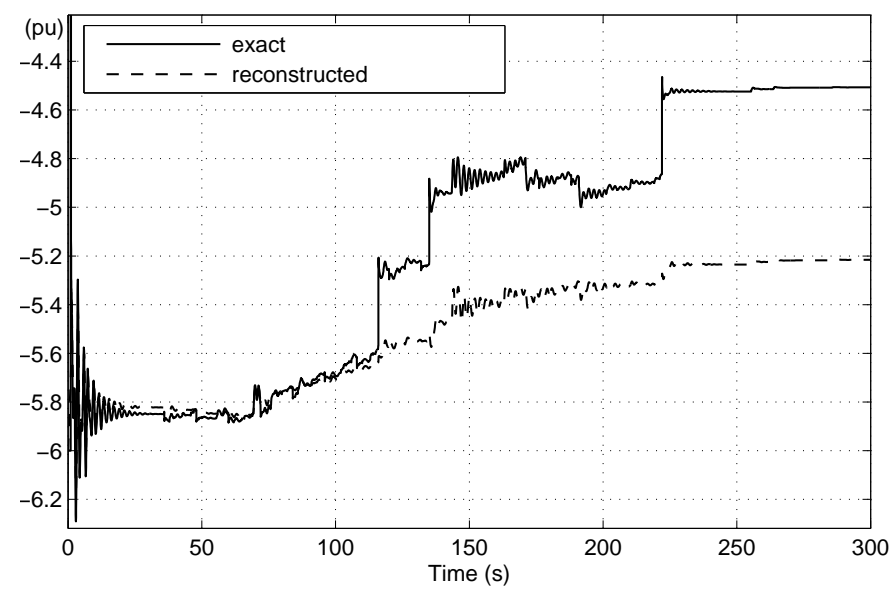

Fig. 10. Exact and reconstructed active power at bus 1041; reconstruction using the method of Ref. [1] (PMU configuration No. 1)

do not carry enough information to detect the jump in system operating conditions. This results in reconstruction errors which can be seen from $t=116 \mathrm{~s}$, when the first load shedding takes place. On the contrary, comparing with Fig. 8 shows 


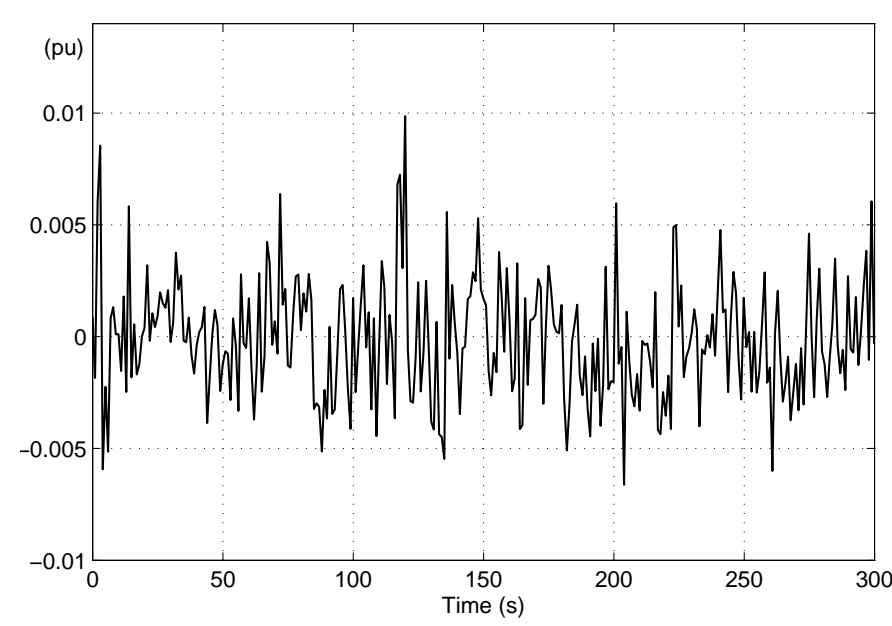

Fig. 11. Error on reconstructed voltage magnitude at bus 1041 (PMU configuration No. 1)

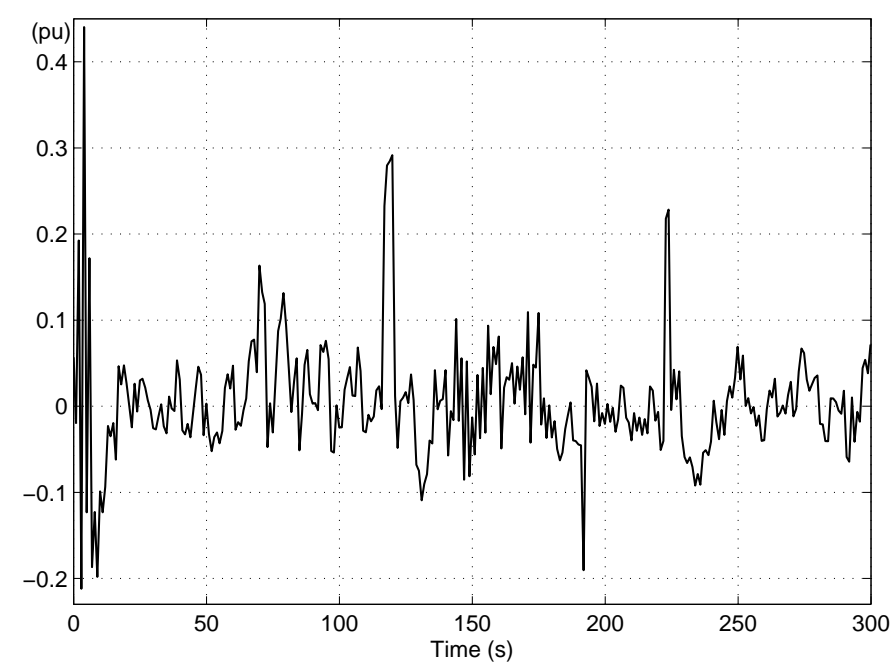

Fig. 12. Error on reconstructed active power of load at bus 1041 (PMU configuration No. 1)

that the method proposed in this paper takes advantage of the information provided by SCADA measurements (in particular the power flow measurement on line 1043-1041), and renders the above mentioned jumps.

For a closer look at reconstruction errors, Figs. 11, 12 and 13 show the difference between the exact and reconstructed values, for the same three outputs. The error peaks in Figs. 12 and 13 correspond to respectively the initial fault, the load curtailments and, to a lower extent, a nearby generator being switched under field current limit by its OEL. These larger errors are caused by inconsistencies of the non-synchronized SCADA measurements. On the other hand, the plots show that the higher errors take place for short durations only.

While from $t=225 \mathrm{~s}$, the system almost reaches steady state, Figs. 12 and 13 show that the reconstruction errors do not vanish. They persist due to the noise affecting SCADA and PMU data. This is confirmed by Fig. 14, which shows

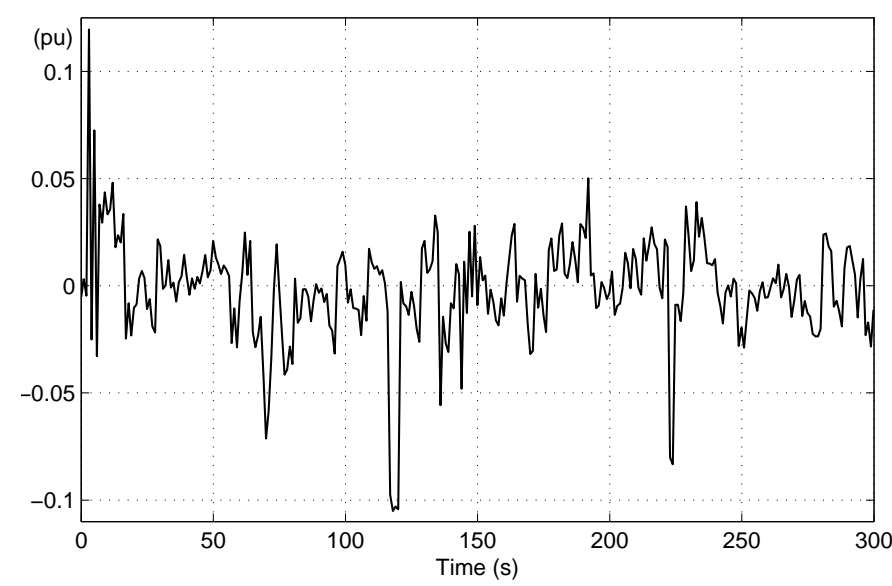

Fig. 13. Error on reconstructed active power flow in line 1043-1041 (PMU configuration No. 1)

(with dashed line) the reconstructed active power flow in line 1043-1041 in the absence of measurement noise on PMU and SCADA measurements. After each significant transient, the reconstructed evolution tends very quickly to the exact one.

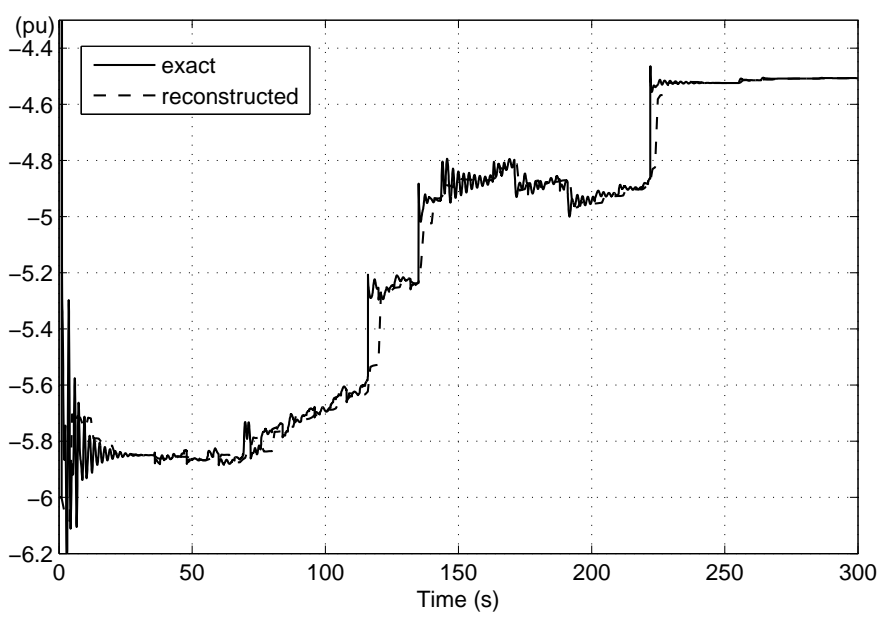

Fig. 14. Exact and reconstructed active power at bus 1041 (PMU configuration No. 1) in the absence of measurement noise

The abnormal reconstructed states can be detected through the index $J(\hat{\boldsymbol{x}})=\sum_{i=1}^{m}\left[\frac{z_{i}-h_{i}(\hat{\boldsymbol{x}})}{\sigma_{i}}\right]^{2}$ where $m$ is the number of measurements and $\hat{\boldsymbol{x}}$ the reconstructed state. It is well known from WLS estimation theory [18] that, if measurement noises have normal distribution, the random variable $J(\hat{\boldsymbol{x}})$ obeys a chi-square distribution with $m-n$ degrees of freedom, where $n$ is the number of components of $\boldsymbol{x}$. For the system being simulated, $m=154+24+20=198$ and $n=2 \times 52=104$, thus $m-n=94$. Assuming a false alarm probability of $1 \%$, the statistical threshold on $J(\hat{\boldsymbol{x}})$ is 128.8 .

The values of $J(\hat{\boldsymbol{x}})$ of the successive reconstructions are shown in Figs. 15 and 16. Figure 15 clearly shows that the states reconstructed immediately after the initial disturbance are unreliable; state reconstruction just after a fault is not appropriate. However, no later than 10 seconds after, $J(\hat{x})$ 


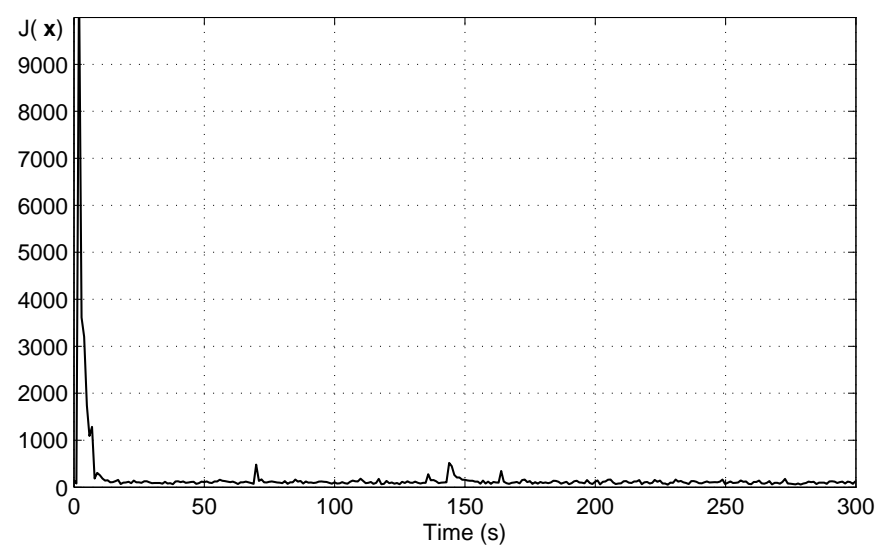

Fig. 15. Values of $J(\hat{\boldsymbol{x}})$ of successive reconstructions

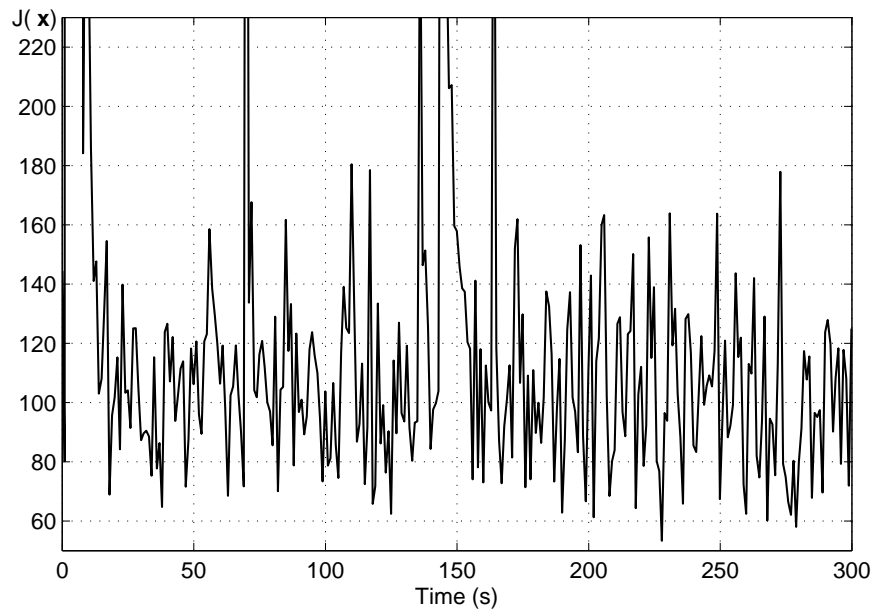

Fig. 16. Values of $J(\hat{\boldsymbol{x}})$ of successive reconstructions (smaller $y$ range)

recovers normal values. It is seen from Fig. 16 that $J(\hat{x})$ crosses the 128.8 threshold rather often. This is an indication that either the system state changes too rapidly for the rate of SCADA measurement update (admittedly, the system is much stressed in this test case!) or the standard deviations assigned to SCADA and pseudo-measurements in $\boldsymbol{W}$ should be somewhat increased.

The second row in Table II shows the mean and standard deviations of the reconstruction errors, after discarding the reconstructed states with $J(\hat{\boldsymbol{x}})>200$. The mean errors are almost negligible (unbiased estimates). For the active power flow in line 1043-1041, the standard deviation of the reconstruction error is a bit more than twice the standard deviation of the measurement noise.

\section{PMU configuration 2}

Figures 17, 18 and 19 show the reconstruction errors, for the same three outputs, when PMU configuration No. 2 is used. The higher accuracy brought by the synchrophasor measurements located closer to bus 1041 is quite visible, especially for the power flow in line 1043-1041. This line is
TABLE II

MEAN AND STANDARD DEVIATION OF RECONSTRUCTION ERRORS (FOR STATES WITH $J(\hat{\boldsymbol{x}}) \leq 200$ )

\begin{tabular}{|c|c|c|c|c|c|c|}
\hline \multirow[t]{2}{*}{$\begin{array}{c}\text { PMU } \\
\text { config. }\end{array}$} & \multicolumn{2}{|c|}{$\begin{array}{l}\text { voltage magnitude } \\
\text { at bus } 1041\end{array}$} & \multicolumn{2}{|c|}{$\begin{array}{c}\text { load active power } \\
\text { at bus } 1041\end{array}$} & \multicolumn{2}{|c|}{$\begin{array}{l}\text { active power flow } \\
\text { in line } 1043-1041\end{array}$} \\
\hline & mean & st. dev. & mean & st. dev. & mean & st. dev. \\
\hline No. 1 & -0.00022 & 0.0025 & 0.0046 & 0.0570 & -0.0022 & 0.0220 \\
\hline No. 2 & -0.00018 & 0.0017 & 0.0016 & 0.0200 & -0.0003 & 0.0028 \\
\hline No. 3 & -0.00120 & 0.0035 & 0.0040 & 0.0597 & -0.0030 & 0.0196 \\
\hline
\end{tabular}

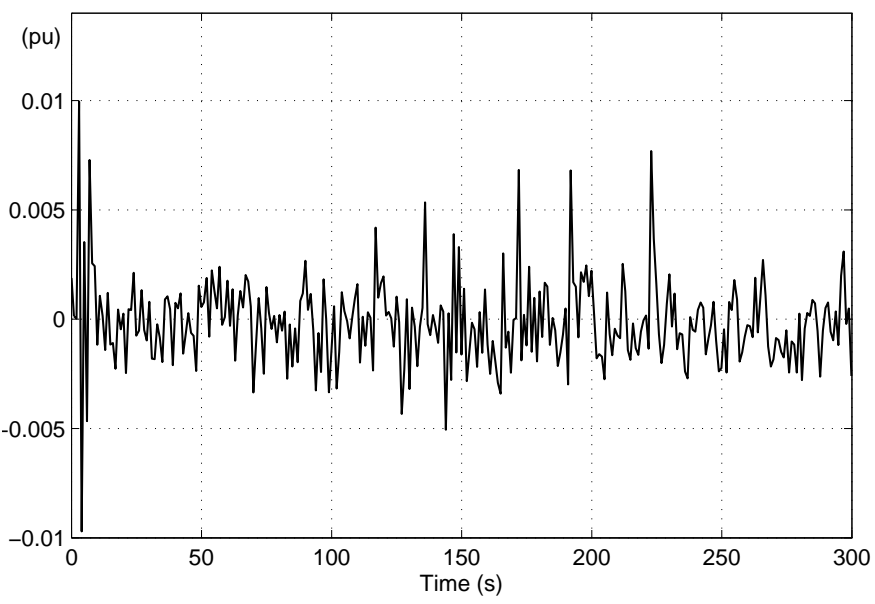

Fig. 17. Error on reconstructed voltage magnitude at bus 1041 (PMU configuration No. 2)

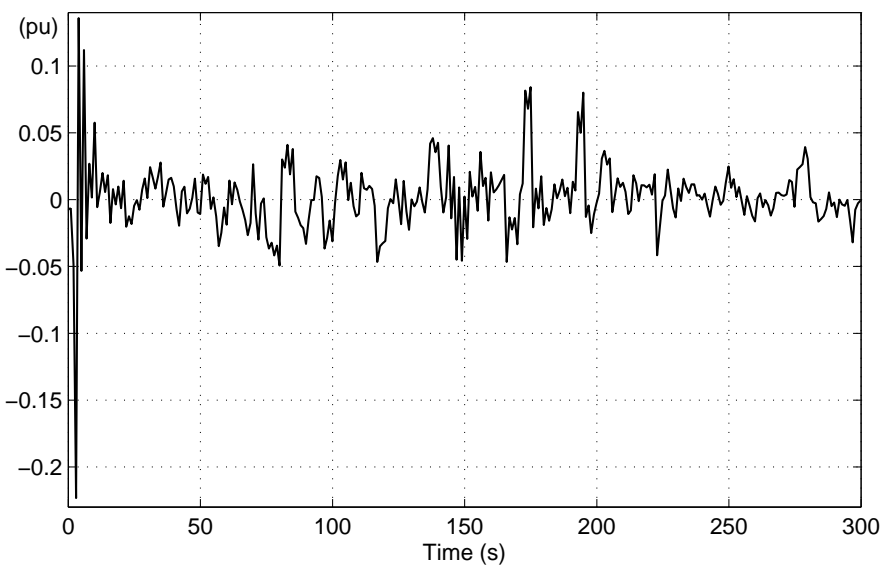

Fig. 18. Error on reconstructed active power of load at bus 1041 (PMU configuration No. 2)

now provided with a synchrophasor measurement of its current instead of a SCADA measurement. The standard deviation of the reconstruction error is also significantly reduced, as can be seen from the third row of Table II.

\section{PMU configuration 3}

The results obtained with PMU configuration No. 3 are given in Figs. 20, 21 and 22 as well as in Table II. In this configuration, PMUs are located farther away from bus 1041. As regards power flows, accuracy is very similar to that obtained with configuration No. 1. In this case, the area near bus 1041 


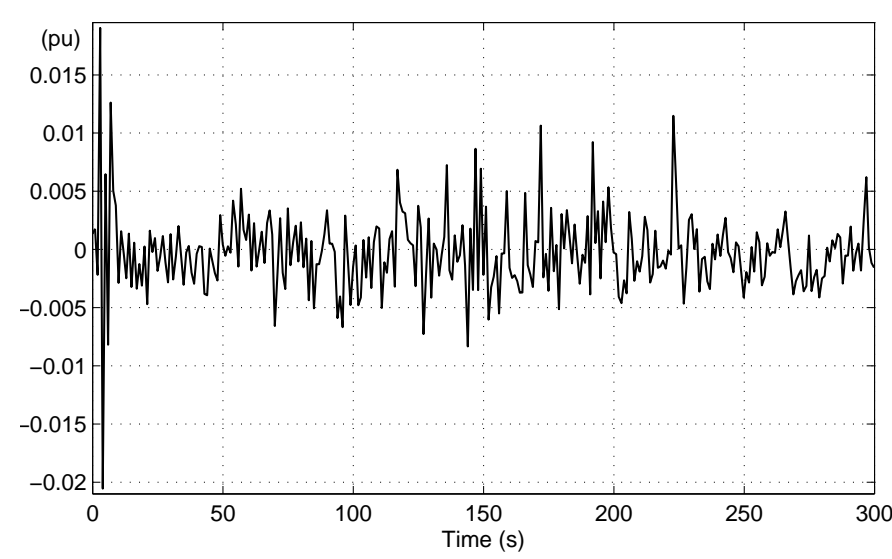

Fig. 19. Error on reconstructed active power flow in line 1043-1041 (PMU configuration No. 2)

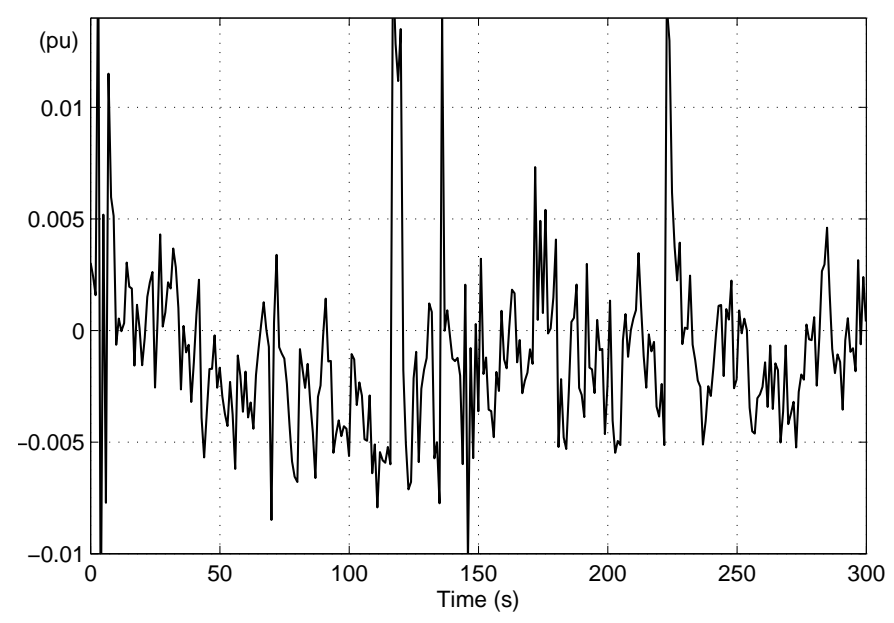

Fig. 20. Error on reconstructed voltage magnitude at bus 1041 (PMU configuration No. 3)

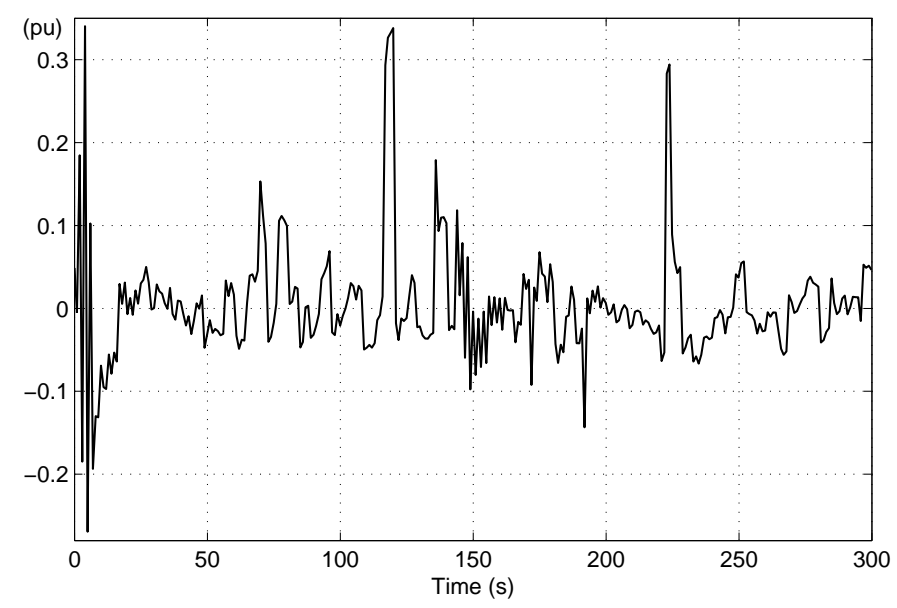

Fig. 21. Error on reconstructed active power of load at bus 1041 (PMU configuration No. 3)

is not provided with PMU, and state reconstruction relies on SCADA measurements essentially.

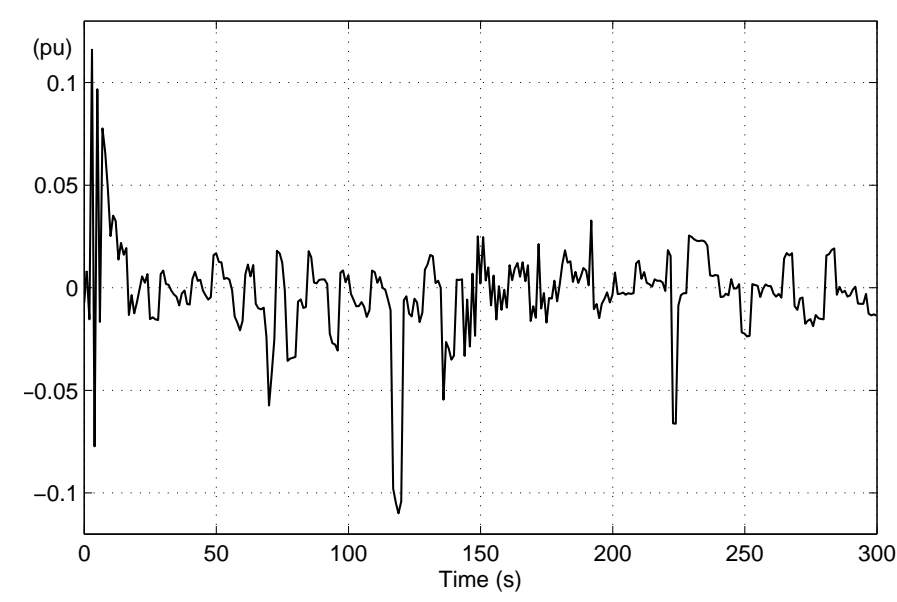

Fig. 22. Error on reconstructed active power flow in line 1043-1041 (PMU configuration No. 3)

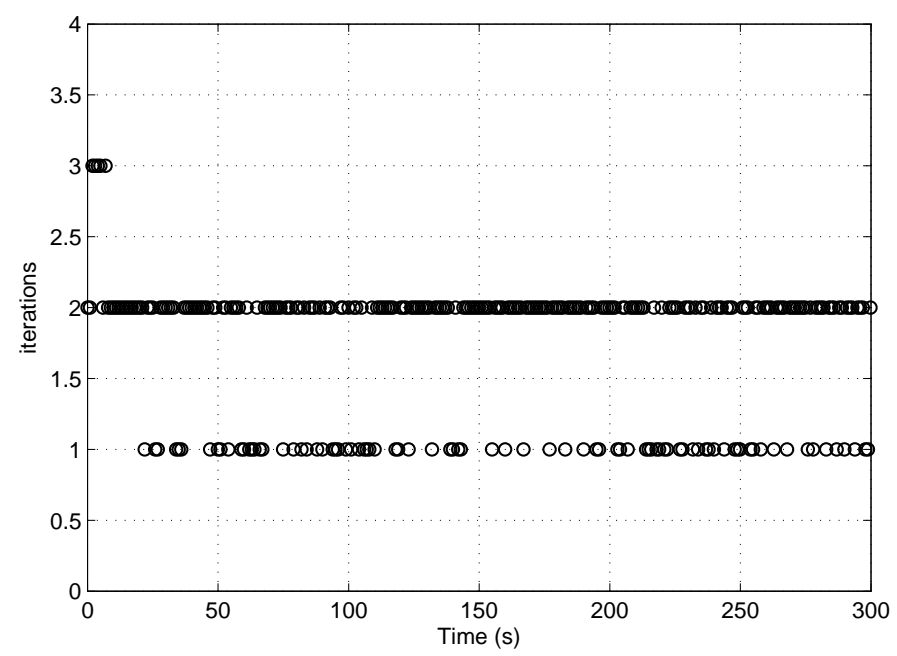

Fig. 23. Number of iterations

\section{Results: performance of optimization procedure}

This section briefly reports on the performance of Hachtel's augmented matrix method. In the tracking mode considered here, for the state reconstruction performed at time $t, \boldsymbol{x}^{0}(t)$ is initialized at $\hat{\boldsymbol{x}}\left(t-T_{r}\right)$, the state vector computed at the previous state reconstruction. The iterations $(6,7)$ are stopped when the absolute change in any rectangular component of the bus voltages is smaller than $0.0001 \mathrm{pu}$.

The number of iterations (i.e. the number of linear systems (6) solved) at each successive state reconstruction is shown in Fig. 23, while a closer look at the first reconstructions is offered in Fig. 24. The number of iterations is remarkably low and stable over time, in spite of the important transients experienced by the system. Even if the initial states are not reliable, as discussed before, Hachtel's method is able to solve the constrained WLS problem very efficiently. 


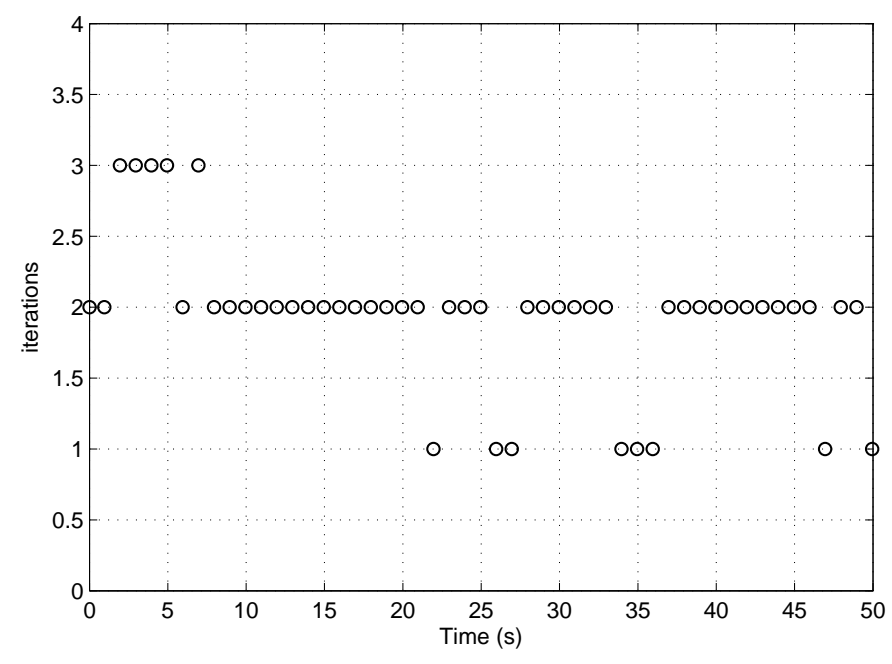

Fig. 24. Number of iterations: detailed view

\section{Conclusion}

A new approach has been proposed to process SCADA and synchronized phasor measurements all together, with the objective of tracking system evolution at a much higher rate than state-of-the-art state estimation, while tackling (through pseudo-measurements obtained in a recursive manner) the unobservability issue caused by the limited number of PMUs.

The following are some of the salient features:

- the method does not require a dynamic model of the system; the bus voltages are tracked without modeling the transition from one state to the next;

- it resorts to a standard WLS estimation, but with a novel way of handling measurements as and when they are received;

- it takes advantage of all available real-time data, even if they are collected at different rates;

- it is scalable: scarce PMU configurations are already exploited, while a higher accuracy will be obtained from richer configurations available in the future.

Among the issues being currently investigated, let us quote:

- exploitation of PMU samples available in between state reconstructions, to improve accuracy and reliability [20];

- exploitation of non-synchronized but time-tagged SCADA measurements;

- a theoretical basis for selecting the weights of respectively synchrophasor, SCADA, and pseudo-measurements;

- diagnosis of unreliable state reconstructions caused by excessive transients;

- identification of persistently erroneous measurements;

- improved numerical solution of the optimization problem (for instance, infrequent updates of Hachtel's matrix);

- state reconstruction of larger systems;

- state reconstruction of a limited region but at higher rates, with the objective of tracking faster dynamics.

\section{References}

[1] M. Glavic and T. Van Cutsem, "Reconstructing and Tracking Network State from a Limited Number of Synchrophasor Measurements," IEEE Trans. Power Syst., vol. 28, no. 2, pp. 1921-1929, May 2013.

[2] A. G. Phadke and J. S. Thorp, Synchronized Phasor Measurements and Their Application, New York: Springer, 2008.

[3] A. Gomez-Exposito, A. Abur, P. Rousseaux, A. de la Villa Jean and C. Gomez-Quiles, "On the Use of PMUs in Power System State Estimation," Proc. 17th Power System Computation Conference (PSCC), Stockholm, Sweden, Aug. 2011.

[4] M. Zhou, V. A. Centeno, J. S. Thorp, and A. G. Phadke, "An Alternative for Including Phasor Measurements in State Eestimators," IEEE Trans. Power Syst., vol. 21, no. 4, pp. 1930-1937, Nov. 2006.

[5] L. Vanfretti, J. H. Chow, S. Sarawgi, and B. Fardanesh, "A PhasorData-Based State Estimator Incorporating Phase Bias Correction," IEEE Trans. Power Syst., vol. 26, no. 1, pp. 111-119, Feb. 2011.

[6] X. Bian, X. R. Li, H. Chen, D. Gan, and J. Qiu, "Joint Estimation of State and Parameters with Synchrophasors-Part I: State Tracking," IEEE Trans. Power Syst., vol. 26, no. 3, pp. 1196-1208, Aug. 2011.

[7] S. Wang, W. Gao, and A. P. S. Meliopoulos, "An Alternative Method for Power System Dynamic State Estimation Based on Unscented Transform," IEEE Trans. Power Syst., Vol. 27, no. 2, pp. 942-950, May 2012.

[8] N. Zhou, Z. Huang, J. Nieplocha, and T. B. Nguyen, "Wide-Area Situational Awareness of Power Grids with Limited Phasor Measurements," Proc. Third International Conference on Critical Infrastructures (CRIS), Alexandria, VA, Sept. 2006.

[9] T. Overbye, P. Sauer, C. DeMarco, B. Lesieutre, and M. Venkatasubramanian, Using PMU Data to Increase Situational Awareness, Power System Engineering Research Center (PSERC) Report 10-16, Sep. 2010.

[10] M. Panteli, P. A. Crossley, D. S. Kirschen, and D. J. Sobajic, "Assessing the Impact of Insufficient Situation Awareness on Power System Operation," IEEE Trans. Power Syst., in Press, 2013.

[11] H. Singh and F. L. Alvarado, "Fast Approximations to LAV Solutions for State Estimation of Power Systems," Proc. 11th Power System Computation Conference (PSCC), Avignon, France, Aug. 1993.

[12] M. Glavic and T. Van Cutsem, "State Reconstruction from Synchronized Phasor Measurements," Proc. IEEE Innovative Smart Grid Technologies (ISGT-Europe), Manchester, UK, Dec. 2011.

[13] A. Debs and R. E. Larson, "A Dynamic State Estimator for Tracking the State of a Power System ," IEEE Trans. Power App. and Syst., vol. PAS-89, pp. 1670-1678, Sep./Oct. 1970.

[14] R. Masiello and F. C. Schweppe, "A Tracking Static State Estimator," IEEE Trans. Power App. and Syst., vol. PAS-90, pp. 1025-1033, Mar./Apr. 1971.

[15] F. F. Wu, K. Moslehi, and A. Bose, "Power System Control Centers: Past, Present, and Future," Proceedings of The IEEE, vol. 93, no. 11, pp. 1890-1908, Nov. 2005.

[16] A Gjelsvik, S. Aam, and L. Holten, "Hachtel's Augmented Matrix Method A Rapid Method Improving Numerical Stability in Power System Static Estimation," IEEE Trans. IEEE Trans. Power App. and Syst., vol. PAS-104, no. 11, pp. 2987-2993, Nov. 1985.

[17] L. Holten, A. Gjelsvik, S. Am, F. F. Wu, and W-H. E. Liu, "Comparison of Different Methods for State Estimation," IEEE Trans. Power Syst., vol. 3, no. 4, pp. 1798-1806, Nov. 1988.

[18] A. Abur and A. Gomez Exposito, Power System State Estimation: Theory and Implementation, Marcel Dekker Inc., New York-Basel, 2004.

[19] T. Van Cutsem, "Description, Modeling and Simulation Results of a Test System for Voltage Stability Analysis", Internal report, University of Liège, 2013, available at http://hdl.handle.net/2268/141234

[20] Q. Zhang, Y. Chakhchouk, V. Vittal, G. T. Heydt, N. Logic, and S. Sturgill, "Impact of PMU Measurement Buffer Length on State Estimation and its Optimization," IEEE Trans. Power Syst., vol. 28, no. 2, pp. 1657-1665, May 2013. 